wissen kompakt 2012 $\cdot 6: 3$

DOI 10.1007/s11838-011-0131-6

(c) Springer-Verlag und Freier Verband

Deutscher Zahnärzte e.V. 2012
4 Dr. med. dent. Norbert Grosse

wissen kompakt konnte sich über viele Jahre stolz und glücklich schätzen, Norbert Grosse als koordinierenden Schriftleiter zu haben - mit dem Erscheinen dieser Ausgabe geht diese lange und außergewöhnlich erfolgreiche Zusammenarbeit zu Ende.

Norbert Grosse hatte die Position als koordinierender Schriftleiter von wissen kompakt bereits seit der ersten Stunde, der Gründung im Jahr 2007, inne. Als Vorsitzender der Akademie Praxis und Wissenschaft (APW) in der Deutschen Gesellschaft für Zahn-, Mund- und Kieferheilkunde (DGZMK), Bundesvorstandsmitglied des Freien Verbandes Deutscher Zahnärzte (FVDZ), Kongressleiter der Fortbildungskongresse in Davos und Usedom - um nur einige seiner zahlreichen Engagements zu nennen - war Norbert Grosse der Fortbildung von jeher eng verbunden. Besonders deutlich zeigte sich diese Verbindung in den guten Kontakten, die Dr. Grosse zu zahlreichen renommierten Wissenschaftlern und Praktikern in Deutschland und der Schweiz pflegte und die sich bald als Autoren in wissen kompakt wiederfanden. Ziemlich schnell war klar: Zusammen mit Norbert Grosse

\title{
Verabschiedung von Dr. Norbert Grosse
}

eine zahnmedizinische Fortbildungszeitschrift zu gründen, ist als echter Glücksfall zu werten.

Bereits von Beginn an war es ein besonderes Anliegen von Dr. Grosse, die Lücke zwischen Wissenschaft und Praxis zu schließen und dem Leser aktuelles und gelegentlich auch einmal trockenes Fachwissen übersichtlich und praxisnah aufbereitet zu vermitteln - und das für alle Bereiche der Zahnmedizin!

Norbert Grosse hat mit der Auswahl wissenschaftlicher Themen und deren praxisnaher Aufbereitung wissen kompakt zu einer Publikation gemacht, die aus der modernen zahnmedizinischen Fortbildungslandschaft nicht mehr wegzudenken ist. Dies beweisen nicht nur die vielen positiven Rückmeldungen der Leser, sondern auch die beeindruckende Anzahl regelmäßiger Online-Teilnahmen an den CME-Fortbildungen, die obligater Bestandteil eines jeden Beitrags in wissen kompakt sind.

\section{1) Dr. Grosse war der Fortbildung von jeher eng verbunden}

Die Nachfolge von Dr. Grosse übernimmt nun ein neues koordinierendes Schriftleitergremium, bestehend aus Prof. Dr. Dr. Bilal Al-Nawas (Mainz), Prof. Dr. Bernd Haller (Ulm) und Prof. Dr. Marc Schmitter (Heidelberg), die die Zeitschrift in der gewohnt hohen Qualität weiterführen werden. Flankiert von den bisherigen und weiterhin aktiven Schriftleitern, den Professoren Edelhoff, Eickholz, Löst, Staehle und Wagner, die als wissenschaft- licher Beirat ihre Expertise auf ihrem jeweiligen Fachgebiet einbringen, ist die Kontinuität des praxisrelevanten Zeitschriftenkonzepts gesichert.

Wir danken Herrn Dr. Norbert Grosse ganz herzlich für seine erfolgreiche Arbeit für wissen kompakt und die immer angenehme Zusammenarbeit. Wir alle wünschen ihm und seiner Familie für die $\mathrm{Zu}$ kunft alles erdenklich Gute und, da wir wissen, dass für ihn „Ruhestand“ nicht gleich „Stillstand“ ist, weiterhin viel Erfolg bei seinen zukünftigen Projekten.

Bilal Al-Nawas

Bernd Haller

Marc Schmitter

Daniel Edelhoff

Peter Eickholz

Claus Löst

Hans Jörg Staehle

Wilfried Wagner

Für den FVDZ:

Karl-Heinz Sundmacher

Bundesvorsitzender des FVDZ

Für Springer Medizin:

Nataša Djordjević

Director Zahnmedizin

Susanne Redeker

Redaktionsleitung wissen kompakt

Sabrina Gabriel

Redaktion wissen kompakt 